\title{
MEDULLOBLASTOMA - CASE PRESENTATION IN AN ADULT PATIENT
}

\author{
Adina Roceanu ${ }^{1}$, Andreea Marinescu ${ }^{2}$, Ovidiu Bajenaru ${ }^{1}$ \\ ${ }^{1}$ Neurology Department, University Emergency Hospital, Bucharest \\ ${ }^{2}$ Radiology Department, University Emergency Hospital, Bucharest
}

\begin{abstract}
The medulloblastoma is an infratentorial, rapidly growing embryonic tumor that arises in the posterior part of the cerebellar vermis and neuroepithelial roof of the fourth ventricle. It is frequent in children relatively uncommon in adults. Medulloblastoma is also named infratentorial primitive neuroectodermal tumor (PNET). We present a case of 28 year old male diagnosed with medulloblastoma in our clinic.
\end{abstract}

Keywords: medulloblastoma, primitive neuroectodermal tumor (PNET)

\section{INTRODUCTION}

Primitive neuroectodermal tumors (PNETs) are typically present as masses in children and adolescents, but rarely in adults.

Primitive neuroectodermal tumors (PNETs) are classified by the World Health Organization as embryonal tumors comprising of undifferentiated or poorly differentiated neuroepithelial cells which appear to or which have the capacity to differentiate into astrocytic, neuronal, ependymal, muscular, or melanocytic lines (1).

PNETs involving the central nervous system can be classified as supratentorial tumors (pineoblastomas) and infratentorial tumors (medulloblastomas).

Central nervous system PNETs are typically found in children or adolescents and very rarely in adults, with less than 100 cases reported in the literature to date. $(4,5)$

The medulloblastoma is an infratentorial, rapidly growing embryonic tumor that arises in the posterior part of the cerebellar vermis and neuroepithelial roof of the fourth ventricle. It is frequent in children relatively uncommon in adults. Medulloblastoma is also named infratentorial primitive neuroectodermal tumor (PNET).
The current view is that the tumor originates from "stem cells" that have not maturate and differentiate, but they have the capacity to divide indefinitely. (6)

Signs and symptoms are mainly due to secondary increased intracranial pressure due to blockage of the fourth ventricle (headache, vomits, and frequent falls in children, diplopia, and strabismus due to VI nerve palsy, dizziness, and nystagmus) and are usually present with few months before diagnosis is made.

PNET tumors of the brain are invasive and rapidly growing tumors that, unlike most brain tumors, spread through the cerebrospinal fluid (CSF) and frequently metastasize to different locations in the brain and spine.

The tumor is characterized by distinctive high signal on both T1 and T2-weighted MRI, with heterogeneous enhancement and typical location adjacent to and extension into the fourth ventricle.

\section{CASE PRESENTATION}

We present the case of a 28 years old male who accused infrequently mild short episodes of headache, without vomiting and mild short episodes of 


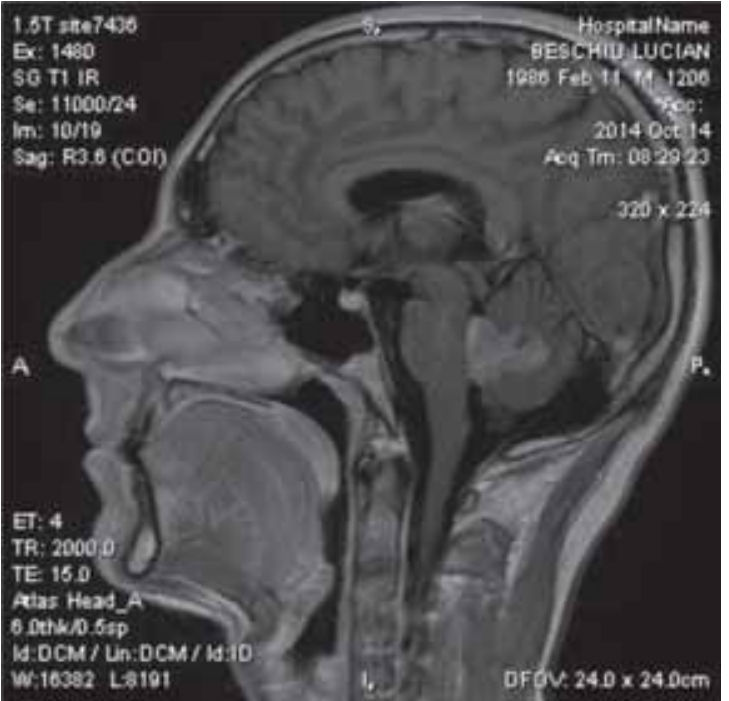

FIGURE 1. Medulloblastoma - MRI sagittal - slight compression of the brainstem
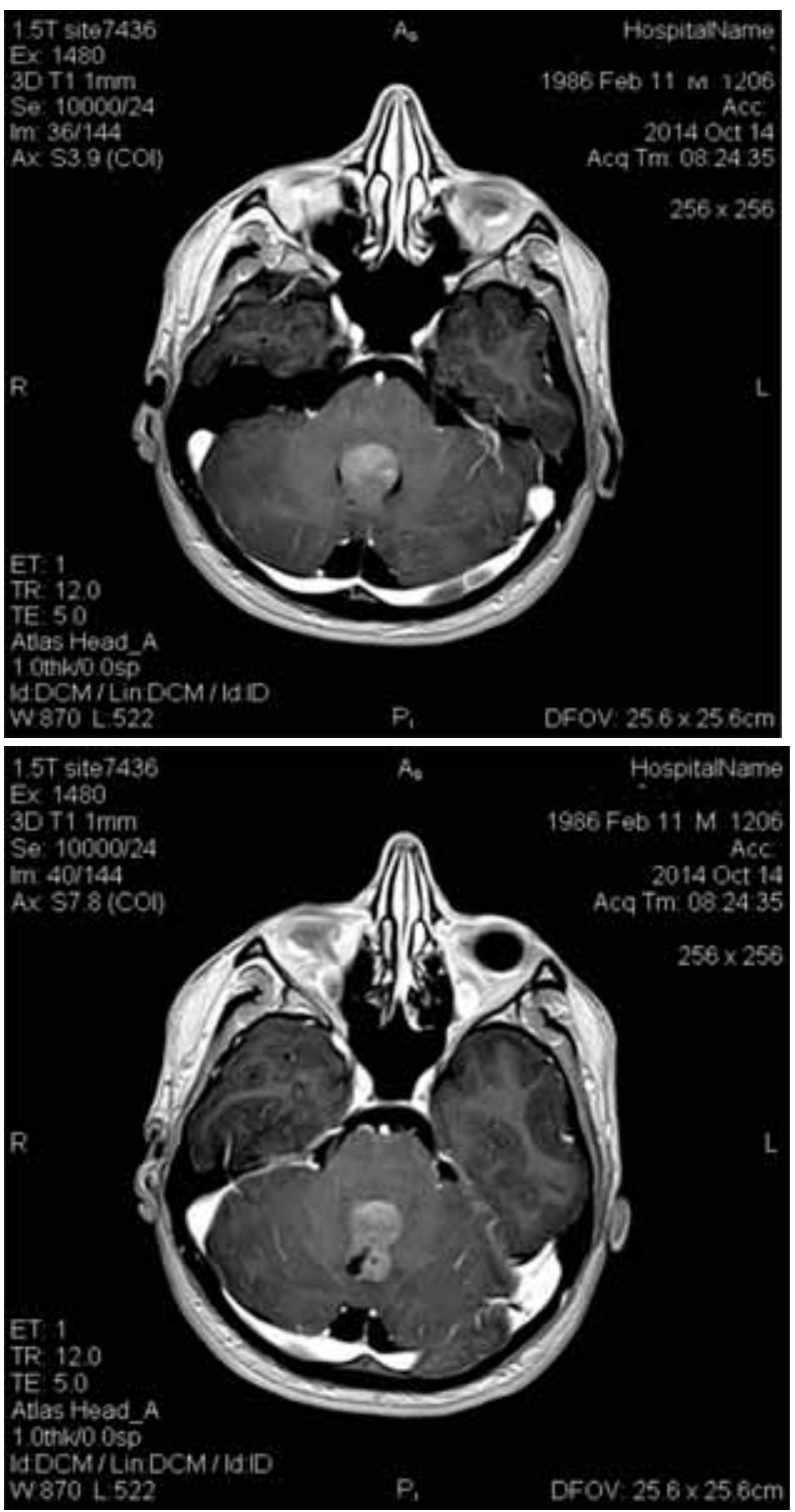

FIGURE 2, FIGURE 3. Medulloblastoma - MRI axial involvement of cerebellar vermis, obliteration of the fourth ventricle dizziness after Valsalva maneuver, started from 1 month ago.

The objective neurological examination didn't disclose cranial nerve palsies or motor, sensory deficits, or impairment of limb coordination. The ENT examination was also normal.

Cerebral IRM disclosed a lobulated, contrastenhancing tumor with cystic areas, projected in the fourth ventricle, with $28 \mathrm{~mm}$ antero-posterior, 18 $\mathrm{mm}$ transversal and $23 \mathrm{~mm}$ cranio-caudal dimensions, with distension of Sylvius apeduct and ventricles I, II, III, without transependimal resorption, suggestive for medulloblastoma. (Fig. 1, 2, 3)

The patient had a maximal resection of the tumor. Pathologic diagnosis confirmed the existence of cerebellar medullovblastoma. Because PNET has high potential to recur and to spread, in addiction chemotherapy and radiotherapy of entire cerebrospinal axis.

\section{CONCLUSION}

The particularity of our case is that medulloblastoma was found in an adult patient with minimal neurological symptoms of recent onset, in contrast with impressive neuroimaging findings.

Due to the low incidence of PNETs in the adult population, there are no evidence-based guidelines for treatment recommended at present $(7,8)$.

\section{REFERENCES}

1. Ohba S., Yoshida K., Hirose Y., Ikeda E., Kawase T. - A supratentorial primitive neuroectodermal tumor in an adult: a case report and review of the literature. J Neurooncol 2008; 86:217-224

2. Brandes A.A., Franceschi E., Tosoni A., Blatt V., Ermani M. Long-term results of a prospective study on the treatment of medulloblastoma in adults. Cancer 2007; 110:2035-2041

3. Brandes A.A., Franceschi E., Tosoni A., Reni M., Gatta G., Vecht C., Kortmann R.D. - Adult neuroectodermal tumors of posterior fossa (medulloblastoma) and of supratentorial sites (stPNET). Crit Rev Oncol Hematol 2009; 71:165-179

4. Lawandy S., Hariri O.R., Miulli D.E., Amin J., Minasian T., Gupta R.K., Siddiqi J. - Supratentorial primitive neuroectodermal tumor in an adult: a case report and review of literature. J Med Case Rep 2012; 6:361

5. Ellis J.A., Rothrock R.J., Moise G., McCormick P.C. II, Tanji K., Canoll P., Kaiser M.G., McCormick P.C. - Primitive neuroectodermal tumors of the spine: a comprehensive review with illustrative clinical cases. Neurosurg Focus 2011; 30:E1

6. Ropper A.H., Brown R.H. - Adams and Victor's Principles of Neurology, The McGraw-Hill Companies, Inc. 2009

7. Kumar L.P., Ahmed S.F., Deepa J., Moinca I., Suresh P., Naidu K.V.J.R. - "Medulloblastoma: A common pediatric tumor: Prognostic factors and predictors of outcome", Asian J Neurosurg. 2015 Jan-Mar; 10(1):50., doi: 10.4103/1793-5482.151516, PMCID: PMC435263

8. Garcia-Armengol R., Guillén-Quesada A., Costa-Clarà J.M. - "Meduloblastoma: infrequent onset in local recurrence", Neurocirugia (Astur). 2011 Oct; 22(5):453-5; discussion 456 